Check for updates

Cite this: RSC Adv., 2021, 11, 27298

\section{Architecture of a multi-channel and easy-to-make microfluidic paper-based colorimetric device $(\mu P C D)$ towards selective and sensitive recognition of uric acid by AuNPs: an innovative portable tool for the rapid and low-cost identification of clinically relevant biomolecules $\uparrow$}

\author{
Fatemeh Farshchi, ${ }^{\text {ab }}$ Arezoo Saadati, ${ }^{c}$ Mohammad Hasanzadeh (D) *bd \\ and Farzad Seidi (iD) *a
}

\begin{abstract}
Uric acid (UA) is the end product of purine metabolism. Uric acid is usually excreted in the urine, but its abnormal increase and toxic amount can lead to diseases such as gout, hyperuricemia, Lesch-Nyhan syndrome, and cardiovascular disease. On the other hand, UA reduction can lead to neurodegenerative diseases such as sarcoma, glioblastoma, Hodgkin, and etc. Therefore, rapid identification of UA is of great importance. In this work, a simple, portable, inexpensive, and fast microfluidic paper-based colorimetric sensor based on the color change in the presence of UA by using AuNPs was developed. The results can be easily identified with naked eye and further confirmed by UV-vis spectrophotometry. In this method, iron pattern and fiberglass paper were used to construct diagnostic areas and hydrophilic microfluidic channels. We greatly reduced the preparation time of this pattern using a magnet (about three minutes). In this work, four types of nanoparticles with different lower limit of quantification (LLOQ) were used. Linear range of $10^{-6}$ to $10^{-3} \mathrm{M}$ and LLOQ of $10^{-6} \mathrm{M}$ were obtained for the determination of uric acid using AuNPs-CysA as optical probe. Also, by AuNPs as optical probe a linear range of $10^{-4}$ to $10^{-2} \mathrm{M}$ and the obtained LLOQ was $10^{-4} \mathrm{M}$. Finally, by AuNFs as optical probe linear range from $10^{-6}$ to $10^{-2} \mathrm{M}$ and $5 \times 10^{-5}$ to $10^{-2} \mathrm{M}$ along with LLOQ of $10^{-6}$ and $5 \times 10^{-5} \mathrm{M}$, respectively. The designed system successfully studied in human urine samples.
\end{abstract}

Received 20th June 2021 Accepted 3rd August 2021 DOI: $10.1039 / \mathrm{d} 1 \mathrm{ra0} 0464 \mathrm{~g}$ rsc.li/rsc-advances of the legs. ${ }^{3}$ Measurement of uric acid is very important in clinical trials. Diagnosis of uric acid is of particular importance for the diagnosis of syndromes as well as its monitoring in patients undergoing chemotherapy or radiation therapy. ${ }^{4,5}$ The standard concentration of UA in urine is 1.4 to $4.4 \mathrm{mM}$ and in serum 0.24 to $0.52 \mathrm{mM}$. Uric acid has usually excreted in the urine, but its abnormal increase can lead to diseases such as gout, hyperuricemia, Lesch-Nyhan syndrome, and cardiovascular disease. ${ }^{6}$ Many of these conditions depend on the diet and lifestyle of patients. ${ }^{7}$ Therefore, early awareness of excessive uric acid highlights the demand for a cheap and simple system to prevent recurrence by changing the diet. On the other hand, reducing uric acid can lead to neurodegenerative diseases such as sarcoma, glioblastoma, Hodgkin, etc. Therefore, rapid identification of UA is of great importance.$^{8-10}$ Common methods for identifying UA were based on the uricase spectrometer, which is the enzymatic oxidation of uric acid by oxygen to produce carbon dioxide, allantoin, and hydrogen peroxide. ${ }^{11}$ Other common methods include chromatography and mass spectrometry. ${ }^{12}$ These methods have drawbacks such as the high ajiangsu Co-Innovation Center for Efficient Processing and Utilization of Forest Resources, International Innovation Center for Forest Chemicals and Materials, Nanjing Forestry University, Nanjing 210037, China. E-mail: f_seidi@njfu.edu.cn

${ }^{b}$ Pharmaceutical Analysis Research Center, Tabriz University of Medical Sciences, Tabriz, Iran. E-mail: hasanzadehm@tbzmed.ac.ir

${ }^{c}$ Drug Applied Research Center, Tabriz University of Medical Sciences, Tabriz, Iran ${ }^{d}$ Nutrition Research Center, Tabriz University of Medical Sciences, Tabriz, Iran

$\dagger$ Electronic supplementary information (ESI) available. See DOI: 10.1039/d1ra04764g 
cost and length of sample preparation. ${ }^{13}$ Also, one of the major drawbacks of these methods is the use of blood serum, which prevents rapid analysis. On the other hand, they require skilled technicians to do the job and require expensive tools. ${ }^{9,14}$ Therefore, one of the urgent needs in the diagnostic field is the design and construction of devices with the study of biochemical processes in the human body for rapid and timely detection of related diseases..$^{15} \mathrm{~A}$ biosensor is a device that uses specific biochemical reactions, usually by specific enzymes, tissues or cells, to electrically detect the chemical elements of the substance, optical, or thermal detection. ${ }^{16}$ These sensors have designed to react with only a specific substance. The result of this reaction is the messages that a microprocessor can analyze. ${ }^{17}$ Sensors due to the unique features such as excellent accuracy and sensitivity have been widely used in UA detection. ${ }^{18}$ Techniques and methods used in the detection of UA include electrochemical methods, ${ }^{19,20}$ fluorometry, ${ }^{21}$ chemiluminescence, ${ }^{22}$ surface Raman spectroscopy and colorimetry. ${ }^{23}$ One of the best methods is colorimetric methods, which in addition to being simple, are also very cheap and can be detect by naked eye. ${ }^{24}$ In recent years, several colorimetric biosensors have designed for UA detection. ${ }^{23,25-27}$ Recently, the integration of advanced biosensors on microfluidic substrates has received much attention. ${ }^{28}$ Microfluidics system is made up of channels with the size of tens to hundreds of micrometers that can analyze small amounts of liquids. Microfluidics has an excellent structure that allows them to be combined with biosensors and increase their application and capability. ${ }^{29}$ These sensors can also be made on paper. ${ }^{30,31}$ Point of care (POC) tests can provide physicians with important and good information, and nowadays these tests are one of the promising points for the use of biosensors. ${ }^{32}$ This system can be a good tool in the rapid and timely diagnosis of diseases, but the design and construction of new tools face serious challenges. ${ }^{33}$ One of the major challenges in microfluid design is its small and portable design and the use of a cheap and accessible substrate without damaging the environment. Nowadays, paper-based sensors have attracted the attention of researchers due to their low cost, ease of movement and use, and great biocompatibility. ${ }^{34}$ These sensors are a great launching pad for the simultaneous detection of multiple analytes. ${ }^{35}$ Due to its hydrophilic nature, paper transfers liquids through its porous structure. ${ }^{36}$ Various methods have used to create and construct a specific pattern that has a hydrophobic and hydrophilic region, such as laser therapy, wax printing, screen printing, and poly dimethyl siloxane (PDMS). ${ }^{37,38}$ One of the best, simplest and cheapest ways to design a pattern and make hydrophobic microchannels on paper is to use liquid paraffin. ${ }^{39}$ Although there is a lot of studies in the use of wax printing technology in the detection of UA, which has good resistance to most chemical compounds, ${ }^{40}$ paraffin is inexpensive and more accessible, so paraffin is a better option. ${ }^{41}$ Paper-based microfluidic analyzers are a new generation of microfluidics that is very simple with high flexibility and can be very suitable as a disposable diagnostic kit. ${ }^{42,43}$ So far, many analytical techniques have been used in paper sensors, fluorescence, electrochemical detection, electrical conductivity, and colorimetry. ${ }^{44}$
Martinez et al. proposed a paper-based biosensor using ELISA, which used micro zone paper for measurement. ${ }^{45}$ Chen et al. designed a microfluidic paper-based colorimetric biosensor that could be detected by the naked eye. ${ }^{46}$ This technique can be employed in smartphones that have high analysis speed. These methods have complexities such as high sample preparation time and cost and low portability. Among these methods, colorimetric detection had higher sensitivity and selectivity in the manufacture of diagnostic kits, and their commercialization is more cost-effective. ${ }^{47}$ Some colorimetric methods use single nanoparticles (gold, platinum, silver, etc.). These nanoparticles have excellent physicochemical properties that can improve the performance of biosensors. ${ }^{48}$ Among nanoparticles, gold nanoparticles (AuNPs) have high optical properties and biocompatibility. ${ }^{49}$ The target molecule causes the accumulation of AuNPs and due to the accumulation of analyte with this nanoparticle, discoloration occurs. ${ }^{50,51}$ As the particle size increases, their catalytic properties decrease, reducing the surface-to-volume ratio. ${ }^{52}$

In this work, we designed a simple, portable, inexpensive and fast microfluidic paper-based colorimetric biosensor based on the color change in the presence of target UA. The results can be easily identified with the naked eye and also proved by UV spectroscopy. Recently, UV-based AuNPs spectroscopy methods for sensitive and selective detection of target species in complex matrices have attracted a lot of attention. ${ }^{53,54}$ AuNPs have a small size, high electron density and excellent catalytic performance, which are the most useful optical properties of fluorescence quenching and surface plasmon intensification (SPR) ${ }^{55}$ According to previous studies, AuNPs can be very useful in identifying uric acid. Additionally, AuNPs are non-toxic and stable against the light. ${ }^{56}$ The AuNPs also have peroxidase-like properties that can catalyze TMB $\left(3,3^{\prime}, 5,5^{\prime}\right.$-tetramethylbenzidine) reduction in the presence of $\mathrm{H}_{2} \mathrm{O}_{2}$ and increase the sensitivity of the resulting dye. ${ }^{57,58}$ These nanoparticles have high stability, high surface area and adjustable catalytic activity ${ }^{59}$ Uric acid changes color through the accumulation of nanoparticles. ${ }^{60}$ Also, liquid paraffin and an iron pattern and fiberglass and TLC papers used to make hydrophilic channels. For easy transfer of the designed pattern on the surface of the paper, paraffin-free paper and paraffin-immersed paper placed in the middle of the magnet and the hot iron pattern. By pressing between the iron and magnet pattern, microfluidic hydro-fluid channels created. This method speeds up pattern making (three minutes) on paper and reduces hand pressure. The low requirements of the stamp tool and the disposable of the microfluidic paper-based device make it possible to use for commercial application in clinical samples analysis based on POC. The designed system is very cheap, simple and portable.

The main innovation of this research work is the simultaneous use of several types of gold nanoparticles and showing their strengths and weaknesses. It is important to point out that, there is no report concerning the effect of size, morphology and other physico-chemical properties of AuNPs on the detection of UA in real samples. So, in this study various type of AuNPs were used for the colorimetric detection of UA in real samples. It is important to point out that, there is no 
comparative study for the evaluation of various type of AuNPs on the detection of $\mathrm{UA}$ by $\mathrm{TMB} / \mathrm{H}_{2} \mathrm{O}_{2}$ reduction process till now. On the other hand, there is no report on the use of paper based microfluid modified by AuNPs for the detection and determination of UA in real samples. Finally, one of the main strengths of this work is the optimization of time and production of very fast and inexpensive paper based microfluidic tool for the onsite detection of UA in biomedical samples using POC.

\section{Experimental}

\subsection{Chemical and reagents}

3,3' $, 5,5^{\prime}$-Tetramethylbenzidine (TMB), hydrogen peroxide, sodium hydroxide, hydrogen chloride, chloroauric acid, cysteamine (CysA), 4-(2-hydroxyethyl)-1-piperazineethanesulfonic acid (HEPES), potassium carbonate, silver nitrate, sodium citrate, sodium borohydride, cetyltrimethylammonium bromide (CTAB), ascorbic acid. TLC paper, fiberglass paper, white paraffin candle.

\subsection{Instrumentation}

The optical examination was performed by UV-VIS spectrophotometer Shimadzu UV-1800 with a resolution of $1 \mathrm{~nm}$. Energy scattering spectroscopy (EDS) was employed to study chemical elements. High-resolution field emission scanning electron microscope (FE-SEM) Hitachi SU8020, Czech with a $3 \mathrm{kV}$ effectual voltage was employed for considering the morphology of electrode surface. Dynamic light scattering (DLS) for zeta potential and size distribution of synthesized nanoparticles was conducted by the zeta potential instrument Malvern Instruments Ltd (Zetasizer Ver. 7.11, MAL1032660, England). AFM was achieved by Nanosurf (AG Gräubernstrasse 12, 4410 Liestal, Switzerland) device.

\subsection{Stock solution preparation}

A mixture of TMB and methanol was prepared at $0.02 \mathrm{M} \mathrm{H}_{2} \mathrm{O}_{2}$ (in a volume ratio of $5: 1$ ) and added with synthesized gold nanoparticles (in a volume ratio of $1: 1$ ). The color of the final solution oxidized to bluish-green. For the control solution, $\mathrm{NaOH}(0.01 \mathrm{~N})$ has prepared with $\mathrm{HCl}(0.01 \mathrm{~N})$, which treated with the combination. Saturated solution of UA $(0.06 \mathrm{M})$ was prepared in $0.01(\mathrm{~N}) \mathrm{NaOH}$ at room temperature. Different concentrations of UA was also prepared using deionized water for further investigation.

\subsection{Universal process for detection of UA}

In this work, different gold nanoparticles with different morphologies were used to detect UA and the color redox reactions of TMB used for UA analysis. The TMB solution is initially colorless and oxidizes to bluish-green in the presence of $\mathrm{H}_{2} \mathrm{O}_{2}$. The gold nanoparticles exhibits peroxidase-like catalytic activity and their color is changed upon mixing with TMB in the presence of $\mathrm{H}_{2} \mathrm{O}_{2}$. After adding UA to the oxidized TMB solution, the TMB was reduced and the colorless solution produced again. Color changes due to TMB redox reaction investigated by UV-Vis spectrophotometry in different solutions (Scheme 1).

\subsection{Preparation of paper-based microfluidic device}

An inexpensive portable microfluidic device was designed and built for colorimetric analysis to examine color changes on paper as well as the effect of concentration on paper. For this purpose, an iron pattern was designed using CorelDRAW software. This pattern has 8 branches that create 8 hydrophilic circular regions that have microfluidic channels $10 \mathrm{~mm}$ long and $30 \mathrm{~mm}$ wide. These channels end in a central area that has a diameter of $10 \mathrm{~mm}$. The overall size of this pattern is $4 \mathrm{~cm}$. Fiberglass and TLC papers were used to create the pattern. Melted paraffin was used to create a hydrophilic environment and transfer the pattern to the paper. Creating a pattern on paper required a lot of pressure and a long time, so for the first time, a very strong magnet was used to prepare the paper bed in the shortest time (about 3 minutes). In the first stage, the metal pattern is placed at a temperature of $150{ }^{\circ} \mathrm{C}$ for 2 minutes, in the second stage, the candle is placed at a temperature of $90{ }^{\circ} \mathrm{C}$ until it melts completely and the paper is immersed in it for 30 seconds, in the third stage after drying complete paraffin paper, paraffin-free paper is placed on the magnet and on it is placed paraffin paper and on it is a hot iron pattern and after 15

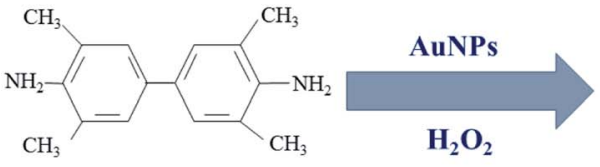

TMB

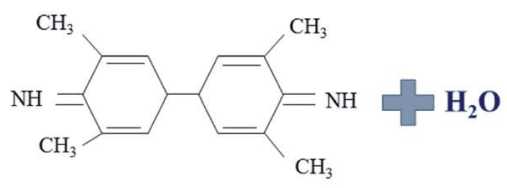

Ox-TMB

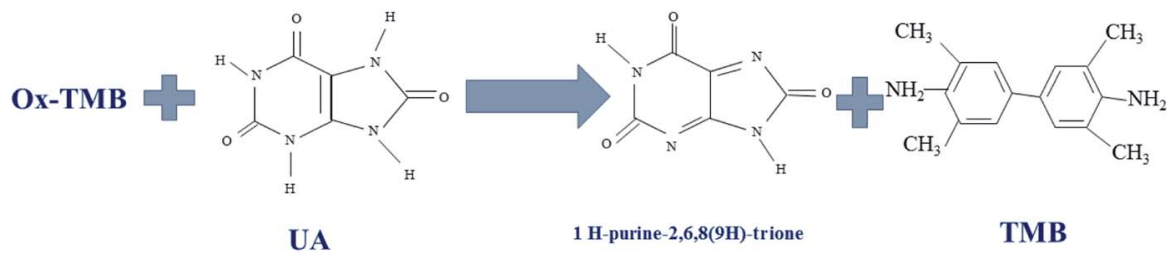

Scheme 1 Schematic illustration of TMB redox reaction. 
seconds we separate it. This allows the candle to penetrate the paper texture and create hydrophilic channels on the paper that are also hydrophobic around it. This system was used to detect uric acid and capillary levels in ducts using a variety of gold nanoparticles (Fig. S1 (see ESI $\dagger$ )) (Scheme 2).

\subsection{Synthesis of nanoparticles (NPs)}

2.6.1. Synthesis of AuNPs-CysA. First, $500 \mu \mathrm{L}$ of $0.5 \mathrm{mM}$ $\mathrm{HAuCl}_{4}$ was magnetically dissolved in $100 \mathrm{~mL}$ of deionized water and heated until boiling point. Then, $5 \mathrm{~mL}$ of sodium citrate $(0.5 \mathrm{M})$ solution was added to the above solution until the yellow color of the solution changed into wine-red, when the created color remained the same, $20 \mu \mathrm{L}$ of CysA $\left(10^{-4} \mathrm{M}\right)$ aqueous solution was added to $10 \mathrm{~mL}$ of above mixture slowly to obtained CysA-AuNPs solution, The final color of the solution was deep red, this solution can be stored at $4{ }^{\circ} \mathrm{C}$ for 2 months.

2.6.2. Synthesis of gold nano-flowers (AuNFs). According to previous studies, $\mathrm{pH}$ can change the morphology of gold nanoparticles. For the aqueous colloidal AuNFs nanoparticle, $50 \mathrm{~mL}$ of $\mathrm{HAuCl}_{4}(50 \mathrm{mM})$ was mixed with $10 \mathrm{~mL}$ of distilled water on a medium speed magnetic stirrer while stirring HEPES solution ( $5 \mathrm{mM}, \mathrm{pH}=6.15$ ) was added to the above solution and stirring was continued for 10 minutes. When the color of the solution began to change, 100 microliters of potassium carbonate was immediately added to the above mixture and the stirrer was continued at a slower speed for up to 1 minute. Then, to investigate the effect of $\mathrm{pH}$ on gold nanoparticles, we repeated this synthesis, this time changing the $\mathrm{pH}$ of HEPES to 4.19 and surprisingly found that the color of the resulting nanoparticles is pink. Change in $\mathrm{pH}$ in the synthesis of gold nanoparticles affects the performance of the adsorption and oxidation process of citrate.

\subsubsection{Gold nano-stars (GNSs) synthesis}

Ag-seeds synthesis. In the first step, $156 \mu \mathrm{L}$ of $\mathrm{AgNO}_{3}(0.0163$ M) and $250 \mu \mathrm{L}$ of 0.005 sodium citrate $\left(\mathrm{Na}_{3} \mathrm{C}_{6} \mathrm{H}_{5} \mathrm{O}_{7}\right)$ were added in 10 ccs of deionized water, respectively. Then $400 \mu \mathrm{L}$ of cold $\mathrm{NaBH}_{4}(0.04 \mathrm{M})$ added to the solution. The yellow color obtained at a distance. This solution kept in a dark place for 2 hours.

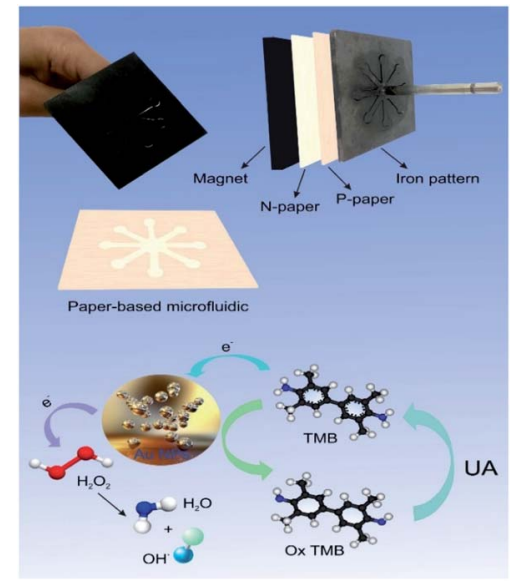

Scheme 2 Schematic illustration of preparation of paper based microfluidic sensor.
Ag growth solution. To prepare gold nano-stars, $0.364 \mathrm{~g}$ of $\mathrm{CTAB}$ was added to deionized water and stirred at $30{ }^{\circ} \mathrm{C}$ to obtain a clear solution, then the temperature turned off and $60 \mu \mathrm{L}$ of $\mathrm{AgNO}_{3}, 100 \mu \mathrm{L}$ of $50 \mathrm{mM} \mathrm{HAuCl}_{4}$ solution and $100 \mu \mathrm{L}$ of $0.08 \mathrm{M}$ ascorbic acid solution added at intervals of 1 minute. After 20 seconds of stirring, $50 \mu \mathrm{L}$ of silver seeds synthesized in the previous step added. The prepared solution was mixed for 15 minutes and then keep at room temperature for 24 hours. The color of the synthesized solution first turns sky blue and then gradually turns brown. After 24 hours, crystals are formed that can be placed at a temperature of 30 degrees and then centrifuged for 15 minutes at $3000 \mathrm{rpm}$ and 5 minutes at $2500 \mathrm{rpm}$, respectively. The colorless supernatant is separated and used.

\section{Characterization}

DLS detection from aggregation analysis provides several advantages and limitations and processes higher sensitivity than conventional colorimeter readings. DLS method, zeta potential analysis used for zeta potential and nanocomposite size distribution. ${ }^{61}$ Also, FE-SEM has been widely used to identify functional nanocomposites in biosensors. ${ }^{62}$ FE-SEM was used in the present work to study the morphology of the synthesized AuNPs. EDX spectroscopy is an analytical method used to study the chemical properties and initial analysis of a sample. ${ }^{63}$ Each element has a unique atomic structure, and this principle is important in EDX, as well as the interaction of a sample and the X-ray excitation source. TEM is a microscopic technique in which an electron beam is transmitted through a sample to create an image. ${ }^{64}$ The image is made of the interaction of the sample and the electrons when the beam is transmitted through the sample. The image is then focused and magnified on an imaging device such as a photographic film layer, a fluorescent screen or a sensor such as a spark plug with a charger. TEM is capable of imaging with significantly higher resolution than light microscopes and is used in several fields of science, such as materials science and nanotechnology. AFM is suitable for observing the structural changes of complex nanostructures in solution and can be said to be the only imaging technique that makes it possible to observe biological compounds without labelling in three-dimensional resolution and at high molecular speed. ${ }^{65}$

\subsection{Characterization of AuNPs-CysA}

As shown in Fig. S2 (see ESI $\dagger$ ), FE-SEM imaging was used to evaluate the morphology of AuNPs-CysA. As it turned out, the spherical nanoparticles were evenly distributed. Preliminary EDX analysis has also used to examine the elements. As shown in Fig. S3 (see ESI $\dagger$ ), the synthesis was successful, and S also demonstrate the successful binding of cysteamine to AuNP. DLS was used to measure the particle size distribution and charge. The various magnifications obtained from TEM were shown in Fig. S4 (see ESI $\dagger$ ). As shown in Fig. S5 (see ESI $\dagger$ ), the particle distribution range of nanoparticles is 10-20 $\mathrm{nm}$. Also, after combining AuNPs-CysA and uric acid DLS was performed. According to Fig. S4C, $\uparrow$ as it is clear, the size of nanoparticles 
has increased to more than $1000 \mathrm{~nm}$. According to the results, it is clear that the interaction of uric acid and nanoprobes has been successfully performed. The zeta potential is negative $(-25$ $\mathrm{mV})$. Saeb et al. have shown that particles with zeta potential values greater than $30 \mathrm{mV}$ or negative more than $-30 \mathrm{mV}$ have good stability.$^{66}$ Also, if the amount of zeta potential increases, the aggregation decreases and the size of the nanoparticles become larger. ${ }^{67}$ Also, the AFM technique was used for 3D imaging. As shown in Fig. S6 (see ESI†). Spherical and white particles of AuNPs are clearly visible on the surface, so this method is a good way to measure the size of nanoparticles in all three dimensions compared to other methods.

\subsection{Characterization of AuNFs}

According to Fig. S7 (see ESI $\dagger$ ), in different synthesis conditions, i.e. different $\mathrm{pH}, \mathrm{AuNFs}$ have the same structure. In this study, the EDC method also used to determine the percentage and frequency of different elements, which was shown in Fig. S8 (see $\mathrm{ESI}_{\dagger}^{\dagger}$ ). Also, to stabilize the successful synthesis and morphology of nanoparticles, TEM imaging was used at different magnifications, as shown in Fig. S9 (see ESI $\dagger$ ), the size of AuNFs is about $40 \mathrm{~nm}$. Based on the obtained results from the DLS technique, although the synthesized AuNFs have the same structure at different pHs, their size was different, as shown in Fig. S10A and B (see ESI $\dagger$ ), when the $\mathrm{pH}$ was 6.15 the average size of AuNFs was $100 \mathrm{~nm}$ while at $\mathrm{pH} 4.19$ the nanoparticle size is $10 \mathrm{~nm}$. It is also shown in Fig. S11C and $\mathrm{D} \dagger$ these nanoparticles, after combining with uric acid, are about 100 times larger in size, confirming the interaction of uric acid and nanoparticles. According to Fig. S10E and F (see ESI $\dagger$ ), the zeta potential of AuNFs at pH 6.15 was $23.9 \mathrm{mV}$ and at $\mathrm{pH} 4.19$ was $31.9 \mathrm{mV}$. Therefore, $\mathrm{pH}$ plays an important role in the size and zeta potential of nanoparticles. The AFM pictures of AuNFs also show in Fig. S11 (see ESI $\dagger$ ). At pH 6.15, the AuNFs provided better images and were spread evenly across the surface, possibly due to the stability of the AuNFs at different pHs.

\subsection{Characterization of GNSs}

Fig. S12 (see ESI $\dagger$ ) shows the FE-SEM images of star-shaped AuNPs that have an average size of 100 to $60 \mathrm{~nm}$ at different magnifications. Also, in TEM imaging, nanoparticles are observed in a completely stellar shape (Fig. S13 (see ESI $\dagger$ )). For the study of smaller nanoparticles, we also used the DLS technique, which shows the micro to the nanoscale, because since the DLS method depends on the interaction of light with particles, it can be used to evaluate the particle size distribution, especially in the range of $2-500 \mathrm{~nm}$. The particle size distribution showed that the average particle size is $20 \mathrm{~nm}$, and after combining with uric acid, the size of nanoparticles has increased to $430 \mathrm{~nm}$ (Fig. S14B (see ESI $\dagger$ )). The zeta potential obtained is $42.6 \mathrm{mV}$, which indicates that this NPs have good stability. Also, the AFM technique was used for 3D imaging. As shown in Fig. S15 (see ESI $\dagger$ ). AuNPs are a white spot peak and are well visible in the figure, so it can be said that this method is a good way to measure the size of nanoparticles in all three dimensions compared to other methods.

\section{Results and discussion}

\subsection{UV-Vis spectroscopic measurements}

Initially, the UV-Vis method was used to monitor color change for detection in the liquid phase. The UV-Vis sub-forums show the synthesized NPs, the bluish-green TMB solution and the UA solutions. No. 1 contains synthesized NPs, no. 2 is a combination of a mixture of UA and $50 \mu \mathrm{L}$ of synthesized AuNPs, no. 3 contains $50 \mu \mathrm{L}$ of bluish-green solution from TMB oxidation and $50 \mu \mathrm{L}$ of synthesized NPs, no. 4 is a combination of no. 3 and a mixture of $\mathrm{NaOH}$ and UA.

4.1.1. AuNPs-CysA. The absorption spectra of synthesized AuNPs-CysA were recorded and analyzed in the presence of UA and dye solution. We also repeated this method to record the stability of the synthesized nanoparticles 1 hour later and to record its images. In AuNPs-CysA synthesis, sodium citrate first reduced and citrate ions act as a stabilizing and reducing agent. In this reaction, citrate ions have negative charges that surround the gold nanoparticles, resulting in a strong electrostatic repulsion that increases the stability of the synthesized gold nanoparticles. The thiol-cysteamine group interacts with the AuNP level via Au-S covalent bonds and stabilizes the amine group, which can react with oxidized TMB and cause discoloration that can also be seen by naked eye. It is attributed to the interaction of CysA-AuNPs with TMB via CysA amino groups at the AuNPs level and UA carboxylic groups, leading to the accumulation of CysA-AuNPs. ${ }^{6,69}$ In this study, AuNP-CysA with $98 \mathrm{~nm}$ in size showed a maximum absorption wavelength at around $520 \mathrm{~nm}$. The color of this nanoparticle is turned into blue in the presence of TMB. Therefore, the UV-Vis absorption spectrum of the synthesized nanoparticles is different, as shown in Fig. S16, $\uparrow$ after adding uric acid due to the increase in nanoparticle size, the adsorption intensity increases sharply to 2.6 and also the adsorption location 637 changes. An increment in the particle dimension or decrement in the interparticle spaces leads to a color change, and a redshift in the maximum absorption wavelength. This significant change attributed to the difference between the particle spacing and the particle size of UA and CysA-AuNP. As mentioned previously, because TMB interacts with AuNPs through thiol-gold interactions, negatively charged amine groups have kept unbound on the surface of CysA-AuNP, leading to repulsion between particles induced by electrostatic repulsion of CysA-AuNP negative charges. Therefore, the distance between CysA-AuNP particles may be greater than AuNP. After adding TMB solution to the nanoparticles due to oxidation, the adsorption intensity decreases, but after adding uric acid to the compound again, the adsorption intensity increases. After one hour, the adsorption intensity of the nanoparticles did not change much, which indicates the stability of the nanoparticles. However, the adsorption intensity of uric acid and nanoparticles greatly reduced and a precipitation was formed according to Fig. S16. $\dagger$ Also, the adsorption intensity of nanoparticles in the presence of uric acid and dye solution decreases sharply after 1 hour due to oxidation.

4.1.2. GNSs. The absorption spectra of the synthesized GNS were recorded and analyzed in the presence of UA and colorless 
solution. This nanoparticle is colorless with no absorption intensity. After adding uric acid, due to the increase in the size of nanoparticles and the reduction of interparticle spaces, the wavelength increases sharply and reaches $3 \mathrm{~nm}$. There is no change o the color of optical probe after addition of TMB. The reason of happen is associated to no redox behaviour of TMB in the presence of this type of AuNPs. In the final composition (4) the adsorption intensity has increased to $3 \mathrm{~nm}$, due to the presence of UA and increasing the size of nanoparticles. We also repeated this method to record the stability of the synthesized nanoparticles 1 hour later and to record its images. Its absorption spectrum is seen only in the presence of UA, which is not stable at all after 1 hour. In short, this nanoparticle is not suitable for the preparation of diagnostic kits, because it does not show any color change and other nanoparticles have better performance.

4.1.3. AuNFs. According to previous studies, $\mathrm{pH}$ can change the morphology of gold nanoparticles. ${ }^{70}$ For example, at low concentrations of citrate due to the reduction of $\mathrm{HAuCl}_{4}$, a network and wire-like structure are formed. A study showed by changing the $\mathrm{pH}$, polyhedral oval and spherical nanoparticles have formed that affect the citrate reduction process. ${ }^{71}$ Therefore, changing the $\mathrm{pH}$ affects the oxidation and adverse effects of protons and citrate adsorption on the surface of gold nanoparticles. According to an important study, a change in $\mathrm{pH}$ can affect the electrostatic interaction between particles, which play an important role in the repulsion and gravity of the environment. In the synthesis of gold nanoparticles, a change in $\mathrm{pH}$ affects the reduction performance of citrate. ${ }^{72,73}$ In this work, the absorption spectrum of the synthesized AuNFs repeated to record the stability of the synthesized AuNFs, one hour later and to capture their images. As shown in the figure, the UV-Vis absorption spectra are different from the AuNFs synthesized at $\mathrm{pH}$, and the color of the nanoparticle's changes from red to purple as the $\mathrm{pH}$ changes due to changes in citrate reduction performance. Due to the adverse effect of protons on citrate adsorption and citrate oxidation on the surface of gold nanoparticles. For AuNFs made at $\mathrm{pH}=6.15$ and 4.19, there are two peaks at 550 and $540 \mathrm{~nm}$. In both cases, UA precipitates in the presence of nanoparticles, so the adsorption intensity is high in
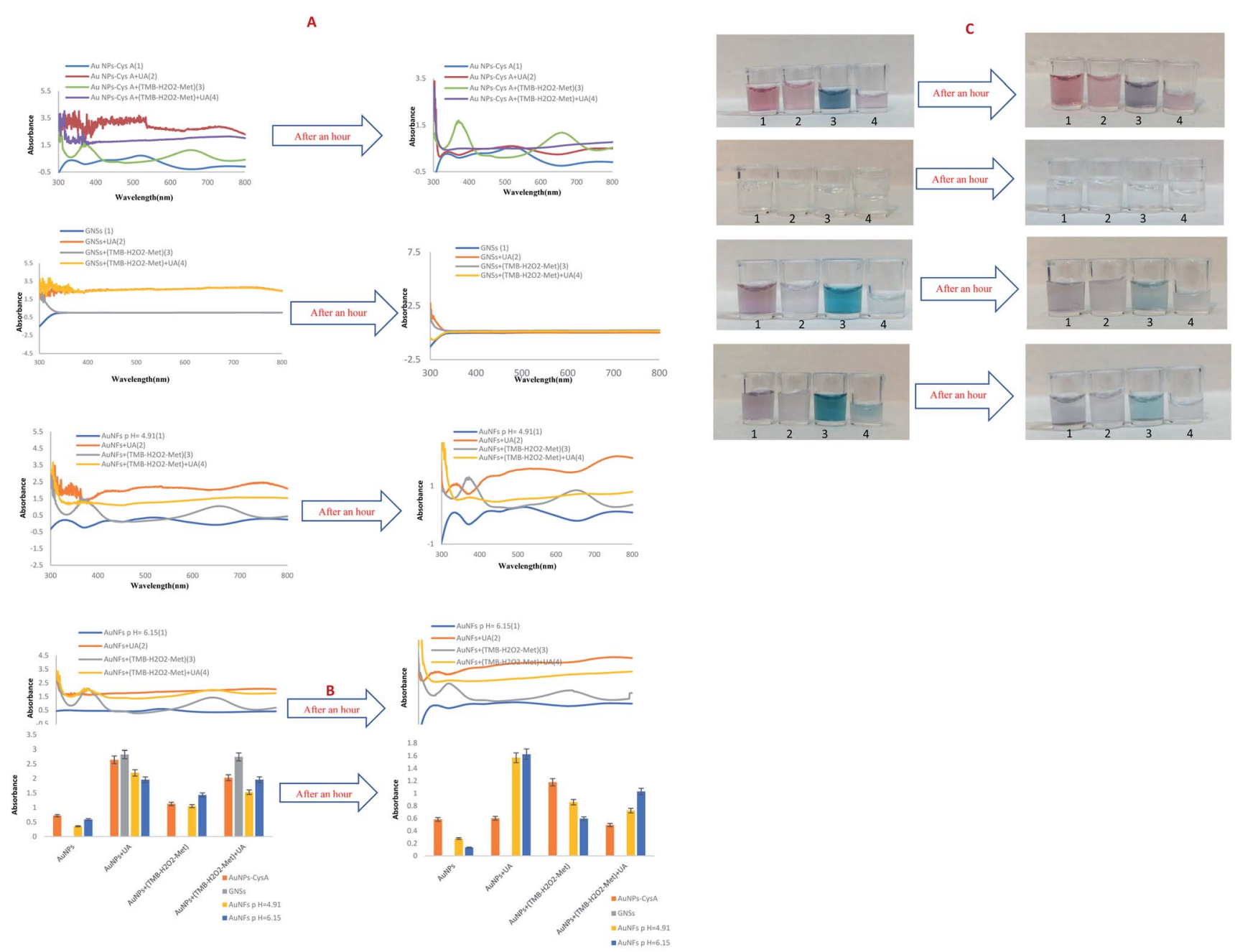

Fig. 1 (A) UV-Vis absorption response of synthesized AuNPs in the mixture of UA and colorless solution and differential UV-Vis absorption response after an hour. (B) Histogram of UV-Vis absorption response of synthesized AuNPs and after an hour. (C) Photographic image of prepared AuNPs in the mixture of UA and colored solution. 
UA-containing compounds. AuNFs synthesized at different pHs show different and maximum UV-Vis spectra. Also, it can be said that electrostatic interactions change the properties of gold nanoparticles. After adding TMB dye solution, AuNFs made in various $\mathrm{pHs},(\mathrm{pH}=6.15$ and 4.91$)$ color change and turn to court blue and light blue, respectively. This also indicates TMB oxidation, as mentioned above, the cause of this different color in different pHs depends on the oxidation of the citrate on the surface of the AuNPs. After adding uric acid to the composition of TMB and AuNFs, TMB reduced and a colorless solution is formed again with uric acid precipitate. The prepared compounds do not have good stability after one hour because the peak intensity decreases and as shown in the pictures, the discoloration of the nanoparticles is quite obvious. Therefore, these nanoparticles do not have good diagnostic power after 1 hour of preparation. The absorption spectrum of the synthesized AuNFs in us was also repeated to record the stability of the synthesized nanoparticles 1 hour later and to capture its images. As shown in the Fig. 1, the UV-Vis absorption spectra are different from the AuNFs synthesized at $\mathrm{pH}$, and the color of the nanoparticle's changes from red to purple as the $\mathrm{pH}$ changes due to changes in citrate reduction performance. The reason for this is the adverse effect of protons on citrate adsorption and citrate oxidation on the surface of AuNFs. For AuNFs made at pHs 6.15 and 4.19, there are two peaks at 550 and $540 \mathrm{~nm}$, respectively. In both cases, UA precipitates in the presence of nanoparticles, so the adsorption intensity is high in UA-containing compounds. AuNFs synthesized at different pHs show different and maximum UV-Vis spectra. Also, it can be said that electrostatic interactions change the properties of gold nanoparticles. After adding TMB dye solution, AuNFs made at pHs 6.15 and 4.91 change color and turn into court blue and light blue, respectively, which also indicates the oxidation of TMB. As mentioned before, the cause of different color change depends on the oxidation of the citrate on the surface of the nanoparticles. After adding uric acid to the composition of TMB and nanoparticles, TMB reduced and a colorless solution is formed again with uric acid precipitate. The prepared compounds do not have good stability after one hour because the peak intensity decreases, as shown in the pictures, the discoloration of the nanoparticles is quite obvious. Therefore, these nanoparticles do not have good diagnostic power after one hour of preparation.

\subsection{Effect of concentration of UA}

The synthesized AuNPs examined in the presence of (AuNPs + TMB $+\mathrm{H}_{2} \mathrm{O}_{2}+$ methanol) solution and different concentrations of UA ( 0.000001 to $0.01 \mathrm{M}$ ) and the prepared solutions used to measure the UV-Vis spectrum. In these experiments, a colorimetric method performed to detect UA, using the peroxidase-like catalytic activity of AuNPs. As shown in Fig. S16-S19 (see ESI $\dagger$ ), as the color intensity of the solution decreases, the absorption intensity also decreases, which is directly visible to the naked eye. There is also a linear relationship between UV-Vis absorption and uric acid concentration. The regression equation has also plotted for each of the nanoparticles, which listed separately in Table S1 (see ESI $\dagger$ ) of the range of uric acid and LLOQ.

As shown in Table 1, although, AuNFs ( $\mathrm{pH}=4.91)$ and AuNPsCysA have the best LLOQ, which indicates a successful design of colorimetric assay, AuNFs ( $\mathrm{pH}=4.91)$ has better performance and linear range $\left(10^{-6}\right.$ to $\left.10^{-2} \mathrm{M}\right)$. The LLOQ of colorimetric biosensor based on AuNFs at $\mathrm{pH}=6.15$ for detection of UA was $5 \times 10^{-5} \mathrm{M}$ and the linear range was from $5 \times 10^{-5}$ to $10^{-2} \mathrm{M}$. GNSs has not good presentation and their UV-Vis absorption was near zero. These nanoparticle is not suitable for the preparation of diagnostic kits, because it does not show any color change and other nanoparticles have better performance. Concentrations less than $10^{-4} \mathrm{M}$ could not be detected and the adsorption intensity was zero at low concentrations. As shown in the images above, the intensity of adsorption also decreases with decreasing concentration. So, in a nutshell, we can say that AuNFs $(\mathrm{pH}=4.91)$ is the most successful nanoparticle in this study (Table 1).

According to Table 1, the designed system has a high diagnostic limit and is much simpler and cheaper compared to other studies, its detection and preparation time is much lower than other proposed systems. One of the most important features of the designed sensor is its portability, which can be used as a simple and inexpensive diagnostic kit for easier use by patients. Based on the obtained results, the dynamic recognition range is wider than these literature values. So, it is expected the prepared sensor paves a novel way for subsequent works in biological studies, and environmental safety test.

\subsection{UV-Vis spectroscopic measurements in real sample}

To evaluate the effect of uric acid concentration in the real sample, a healthy urine sample used without any pretreatment.

Table 1 Evolution performance of the prepared sensor for the detection of uric acid in biological specimens

\begin{tabular}{|c|c|c|c|c|}
\hline Analytical technique & Reaction system & Linear range $(\mathrm{M})$ & LLOQ-LOD (M) & Ref. \\
\hline Electrochemical & Nafion/uricase/ferrocene/GCE & $0.5-600 \times 10^{-6}$ & $230 \times 10^{-12}$ & 74 \\
\hline Electrochemical & Uricase/Au-rGO/ITO & $50-800 \times 10^{-6}$ & $7.32 \pm 0.21 \times 10^{-6}$ & 75 \\
\hline Fluorescence/colorimetric & $\mathrm{N}-\mathrm{CQDS} / \mathrm{Ag}$ TNPs- $\mathrm{H}_{2} \mathrm{O}_{2}$ & $0.1-45 \times 10^{-6}$ & $0.05 \times 10^{-6}$ & 76 \\
\hline Electrochemical & $\mathrm{UOx} / \mathrm{Fc} / \mathrm{Cu}_{2} \mathrm{O} / \mathrm{GCE}$ & $0.01-1 \times 10^{-3}$ & $0.0596 \times 10^{-6}$ & 77 \\
\hline Colorimetry & $\mathrm{TMB} / \mathrm{g}-\mathrm{C}_{3} \mathrm{~N}_{4} /$ uricase & $10-100 \times 10^{-6}$ & $8.9 \times 10^{-6}$ & 78 \\
\hline Chemiluminescence & TCPO- $\mathrm{H}_{2} \mathrm{O}_{2}$-rubrene & $10-1000 \times 10^{-6}$ & $5.0 \times 10^{-6}$ & 79 \\
\hline \multirow[t]{4}{*}{ Fluorescence/colorimetric } & AuNPs-CysA/TMB- $\mathrm{H}_{2} \mathrm{O}_{2}$ & $10^{-3}$ to $10^{-6}$ & $10^{-6}$ & \multirow[t]{4}{*}{ This work } \\
\hline & GNSs/TMB- $\mathrm{H}_{2} \mathrm{O}_{2}$ & $10^{-2}$ to $10^{-4}$ & $10^{-4}$ & \\
\hline & AuNFs $\mathrm{pH}=4.91 / \mathrm{TMB}-\mathrm{H}_{2} \mathrm{O}_{2}$ & $10^{-2}$ to $10^{-6}$ & $10^{-6}$ & \\
\hline & AuNFs $\mathrm{pH}=6.15 / \mathrm{TMB}-\mathrm{H}_{2} \mathrm{O}_{2}$ & $10^{-2}$ to $5 \times 10^{-5}$ & $5 \times 10^{-5}$ & \\
\hline
\end{tabular}


First, urine samples combined with known concentrations of UA. In addition, UV-Vis spectrophotometry performed to calculate its improvement and calculated according to the response current with a standard calibration curve. Except for AuNPs-CysA, which decreases with decreasing adsorption intensity, the rest of the nanoparticles increase with decreasing adsorption intensity, depending on the type of reaction (Fig. S20-S23 (see ESI $\dagger$ )).

\section{Paper-based colorimetric detection of uric acid}

Although UV-Vis spectrophotometric results are very suitable for UA detection, their use is limited and can only be used in laboratories. Paper-based microfluidics can be used to apply and fabricate the kit as a tool to detect and determine the effect of UA concentration change. In recent years various sensors have been designed to increase the efficiency and recognition of UA. Microfluidic paper-based sensors have been designed with unique features. Recently, with the rapid development of microfluidics in various fields such as chemistry and life sciences, their integration with sensors promises a new way of identifying different analytes. They have made significant strides in this area ${ }^{80}$ Also, they have great potential in identifying and analyzing various analytes such as UA, which we hope can become a commercial product in the future. Paper-based microfluidic analyzers are a new generation of microfluidics that are very simple and highly flexible. Paper-based microfluidics can be used to apply and fabricate kits as a tool to identify and determine the effect of UA concentration changes in laboratories. ${ }^{30}$ The prepared paper-based template has microchannels that have a capillary flow and the liquid can pass through it easily. So, it has a pump that makes it an attractive layer for the detection of UA. On the other hand, paper is very cheap, available, and portable. All this adds to the benefits of the designed biosensor. The prepared microfluidic paper-based biosensor was made in a small size, which has better advantages compared to the old sensors, such as low material consumption and easy sample transfer. Older systems require complex and multi-stage tools that have limited use in some areas. The designed microfluid can analyze several samples simultaneously. In this work, to evaluate the performance of papers, TLC and fiberglass paper were used, the results showed

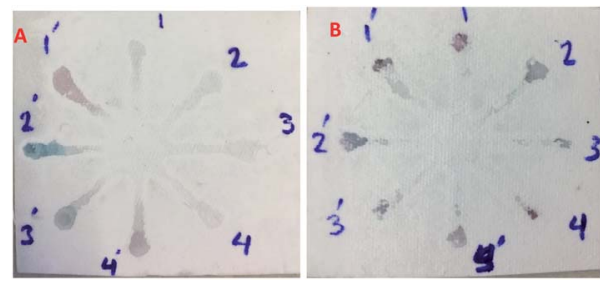

Fig. 2 Photographic images of the TLC microfluidic paper-based calorimetric sensor for reaction systems: (A) GNSs (zone 1-4) and AuNPs-CysA (zone 1'-4'), (B) AuNFs pH $=4.91$ (zone 1-4) and AuNFs $\mathrm{pH}=6.15\left(\right.$ zone $\left.1^{\prime}-4^{\prime}\right)$.
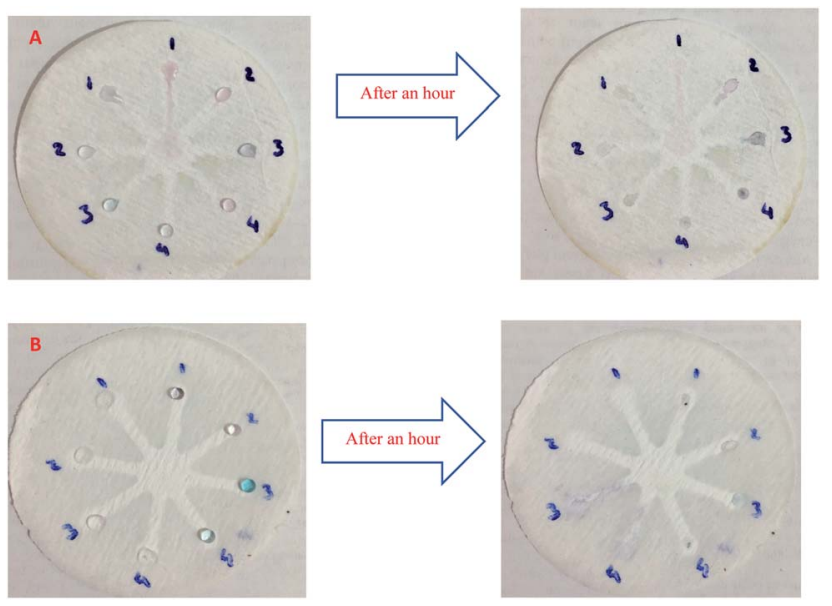

Fig. 3 Photographic images of the fiberglass microfluidic paperbased calorimetric sensor for reaction systems: (A) AuNFs pH $=6.15$ (zone 1-4 left) and AuNPs-CysA (zone 1-4 right), (B) GNSs (zone 1-4 left) and AuNFs ( $\mathrm{pH}=4.91)$ (zone 1-4 left).

that fiberglass paper has less friction due to better flow. So this type of substrate used to investigate the detection of UA and its determination (Fig. 5 and 6) which recorded one hour later fully confirm this claim. Also, to evaluate the effect of UA concentration in the urine sample on the prepared microfluidic substrate, the prepared concentrations placed on the sensing zones of the prepared paper. The results showed that proposed platform can be used as a colorimetric kit for clinical analysis of UA. To check the capillary power of the microchannel, $10 \mu \mathrm{L}$ of UA placed in the center of the prepared microfluidic paperbased sensor and the compounds containing nanoparticles and dye solution were placed in the assay area (Fig. 3).

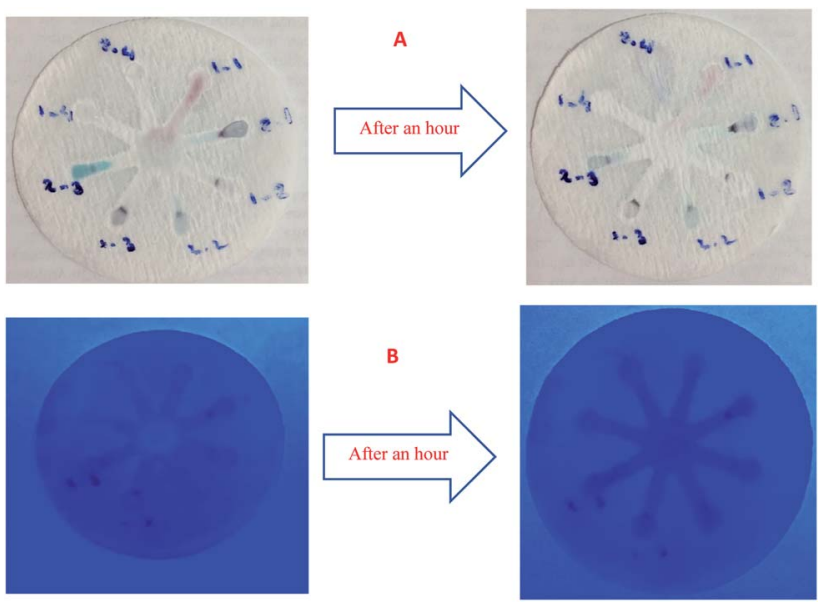

Fig. 4 (A) photographic images of the fiberglass microfluidic paperbased calorimetric sensor for evaluation of capillary: AuNPs-CysA and colored TMB solution and AuNPs-CysA (zone 1-1 and 2-1), AuNFs pH $=6.15$ and colored TMB solution and AuNFs pH $=6.15$ (zone 1-2 and $2-2)$, AuNFs $\mathrm{pH}=4.91$ and colored TMB solution and $\mathrm{AuNFs} \mathrm{pH}=4.91$ (zone 1-3 and 2-3), GNSs and colored TMB solution and GNSs (zone 1-4 and 2-4). (B) Colorimetric detection of UA by capillary method under UV light. 

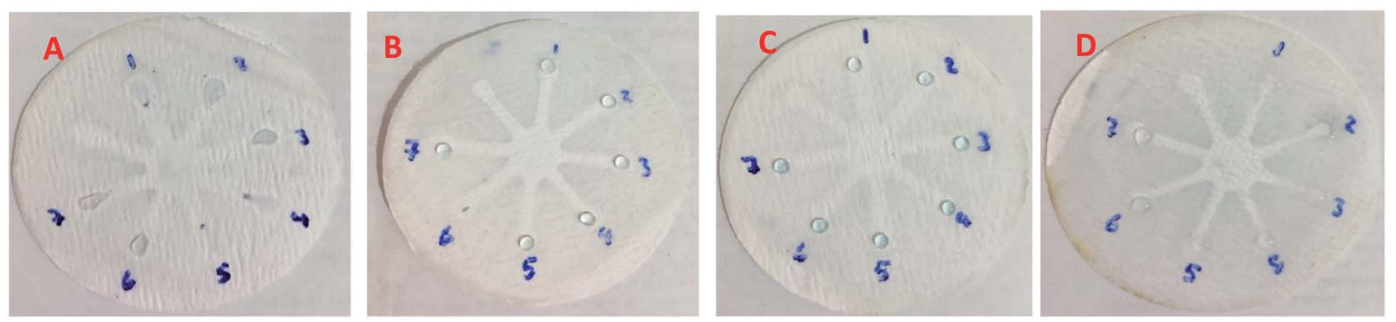

Fig. 5 Photographic images of the fiberglass microfluidic paper-based calorimetric sensor in different concentration of UA: (A) AuNPs-CysA, (B) AuNFs ( $\mathrm{pH}=6.15$ ), (C) AuNFs ( $\mathrm{pH}=4.91$ ), and (D) GNSs. Zones 1 to 7 contain concentrations of 0.00001, 0.00005, 0.0005, 0.0001, 0.005, 0.001, and $0.01 \mathrm{M}$ uric acids, respectively.
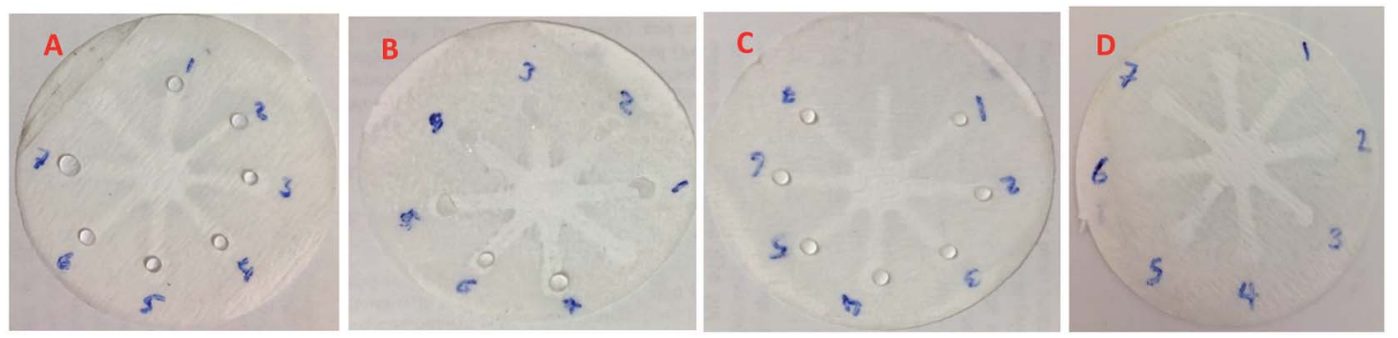

Fig. 6 Photographic images of the fiberglass microfluidic paper-based calorimetric sensor in different concentration of UA in human urine samples: (A) AuNPs-CysA, (B) AuNFs ( $\mathrm{pH}=6.15)$, (C) AuNFs ( $\mathrm{pH}=4.91)$, and (D) GNSs. Zones 1 to 7 contain concentrations of 0.00001, 0.00005, $0.0005,0.0001,0.005,0.001$, and $0.01 \mathrm{M}$ uric acids, respectively.

According to the results, the channels have good pumping power. Hence, low friction and flow in microchannels are appropriate, and over time, reaction systems and uric acid flow into each other inside microfluid channels (Fig. 4). Therefore, this technique can be used to design and fabricate microfluidic paper-based colorimetric sensor to detect glucose, amino acids, and so on (Fig. 2).

\section{Conclusion}

In this study, we developed a facile, portable, inexpensive and fast microfluidic paper-based colorimetric sensor based on the color change in the presence of target UA. The results can be easily identified with the naked eye. In this research work, different type of AuNPs with various morphologies were used to detection of UA and the color change in the redox reaction of $\mathrm{TMB} / \mathrm{H}_{2} \mathrm{O}_{2}$ was used for the analysis UA in real samples. Color changes resulting from the redox reaction were investigated by UV-Vis spectrophotometry in various solutions. The AuNPs which used in this work have high stability, high surface area and adjustable catalytic activity. Also, liquid paraffin and an iron pattern and fiberglass and TLC papers were used to make hydrophilic channels. The employed AuNPs have different lower limit of quantification (LLOQ). AuNPs-CysA has a linear range of $10^{-6}$ to $10^{-3} \mathrm{M}$ and the obtained LLOQ was $10^{-6} \mathrm{M}$. GNSs have a linear range of $10^{-4}$ to $10^{-2} \mathrm{M}$ and the obtained LLOQ was $10^{-4} \mathrm{M}$. AuNFs at pHs of 4.91 and 6.15 have a linear range from $10^{-6}$ to $10^{-2} \mathrm{M}$, and $10^{-2}$ to $5 \times 10^{-5} \mathrm{M}$, LLOQ $10^{-6}$ and $5 \times 10^{-5} \mathrm{M}$, respectively. The AuNFs with $\mathrm{pH}=4.91$ showed the best results. Also, GNSs have not shown suitable results. So, it is not a suitable nanoparticle for the preparation of diagnostic kits. The designed system exhibited excellent performance in detection of UA in real samples such as human urine samples. Therefore, this system has high ability to be applied as a powerful kit in clinical laboratories and has great features to be commercialized.

\section{Conflicts of interest}

There are no conflicts to declare.

\section{Acknowledgements}

We gratefully acknowledge Tabriz University of Medical Sciences for instrumental supporting of this research.

\section{References}

1 G. Glantzounis, E. Tsimoyiannis, A. Kappas and D. Galaris, Curr. Pharm. Des., 2005, 11, 4145-4151.

2 Y. Y. Sautin and R. J. Johnson, Nucleosides, Nucleotides Nucleic Acids, 2008, 27, 608-619.

3 D.-H. Kang, T. Nakagawa, L. Feng, S. Watanabe, L. Han, M. Mazzali, L. Truong, R. Harris and R. J. Johnson, J. Am. Soc. Nephrol., 2002, 13, 2888-2897.

4 N. M. Elsayed, J. M. Nakashima and E. M. Postlethwait, Arch. Biochem. Biophys., 1993, 302, 228-232.

5 R. Archibald, Clin. Chem., 1957, 3, 102-105.

6 A. Leiba, S. Vinker, D. Dinour, E. J. Holtzman and M. Shani, J. Am. Soc. Hypertens., 2015, 9, 600-609. 
7 H. K. Choi and G. Curhan, Curr. Opin. Rheumatol., 2005, 17, 341-345.

8 R. El Ridi and H. Tallima, J. Adv. Res., 2017, 8, 487-493.

9 A. Virdis, S. Masi, E. Casiglia, V. Tikhonoff, A. F. Cicero,

A. Ungar, G. Rivasi, M. Salvetti, C. M. Barbagallo and M. Bombelli, Hypertension, 2020, 75, 302-308.

10 C. King, M. A. Lanaspa, T. Jensen, D. R. Tolan, L. G. SánchezLozada and R. J. Johnson, Uric Acid in Chronic Kidney Disease, 2018, vol. 192, pp. 88-102.

11 K. N. Glazebrook, L. S. Guimarães, N. S. Murthy, D. F. Black, T. Bongartz, N. J. Manek, S. Leng, J. G. Fletcher and C. H. McCollough, Radiology, 2011, 261, 516-524.

12 L. Xu, Y. Shi, S. Zhuang and N. Liu, Oncotarget, 2017, 8, 100852.

13 I. Ramazzina, C. Folli, A. Secchi, R. Berni and R. Percudani, Nat. Chem. Biol., 2006, 2, 144-148.

14 J. Dawson, T. Quinn and M. Walters, Curr. Med. Chem., 2007, 14, 1879-1886.

15 M. L. Muiesan, M. Salvetti, A. Virdis, S. Masi, E. Casiglia, V. Tikhonoff, C. M. Barbagallo, M. Bombelli, A. F. Cicero and M. Cirillo, J. Hypertens., 2021, 39, 62-69.

16 C. Ziegler and W. Göpel, Curr. Opin. Chem. Biol., 1998, 2, 585-591.

17 S. Vigneshvar, C. Sudhakumari, B. Senthilkumaran and H. Prakash, Frontiers in Bioengineering and Biotechnology, 2016, 4, 11.

18 L. Farzin, M. Shamsipur, L. Samandari and S. Sheibani, Microchim. Acta, 2018, 185, 1-25.

19 W. Shi, J. Li, J. Wu, Q. Wei, C. Chen, N. Bao, C. Yu and H. Gu, Anal. Bioanal. Chem., 2020, 412, 7275-7283.

20 X.-J. Huang, H.-S. Im, O. Yarimaga, J.-H. Kim, D.-H. Lee, H.-S. Kim and Y.-K. Choi, J. Phys. Chem. B, 2006, 110, 21850-21856.

21 Y. Liu, H. Li, B. Guo, L. Wei, B. Chen and Y. Zhang, Biosens. Bioelectron., 2017, 91, 734-740.

22 T. Khajvand, M. J. Chaichi and A. H. Colagar, Food Chem., 2015, 173, 514-520.

23 H.-F. Lu, J.-Y. Li, M.-M. Zhang, D. Wu and Q.-L. Zhang, Sens. Actuators, B, 2017, 244, 77-83.

24 N. Alizadeh, S. Ghasemi, A. Salimi, T.-K. Sham and R. Hallaj, Colloids Surf., B, 2020, 195, 111228.

25 A. Badoei-Dalfard, N. Sohrabi, Z. Karami and G. Sargazi, Biosens. Bioelectron., 2019, 141, 111420.

26 Y. He, F. Qi, X. Niu, W. Zhang, X. Zhang and J. Pan, Anal. Chim. Acta, 2018, 1021, 113-120.

27 K. Khachornsakkul and W. Dungchai, RSC Adv., 2020, 10, 24463-24471.

28 N.-T. Nguyen, S. T. Wereley and S. A. M. Shaegh, Fundamentals and applications of microfluidics, Artech House, 2019.

29 G. M. Whitesides, Nature, 2006, 442, 368-373.

30 X. Li, D. R. Ballerini and W. Shen, Biomicrofluidics, 2012, 6, 011301.

31 C. Carrell, A. Kava, M. Nguyen, R. Menger, Z. Munshi, Z. Call, M. Nussbaum and C. Henry, Microelectron. Eng., 2019, 206, 45-54.

32 S. K. Sia and L. J. Kricka, Lab Chip, 2008, 8, 1982-1983.
33 S. Sharma, J. Zapatero-Rodríguez, P. Estrela and R. O'Kennedy, Biosensors, 2015, 5, 577-601.

34 J. Sun, Y. Xianyu and X. Jiang, Chem. Soc. Rev., 2014, 43, 6239-6253.

35 F. Farshchi, A. Saadati and M. Hasanzadeh, Anal. Methods, 2020, 12, 4759-4768.

36 B. Liu, D. Du, X. Hua, X. Y. Yu and Y. Lin, Electroanalysis, 2014, 26, 1214-1223.

37 Y. He, Y. Wu, J. z. Fu, Q. Gao and J. j. Qiu, Electroanalysis, 2016, 28, 1658-1678.

38 G. Luka, A. Ahmadi, H. Najjaran, E. Alocilja, M. DeRosa, K. Wolthers, A. Malki, H. Aziz, A. Althani and M. Hoorfar, Sensors, 2015, 15, 30011-30031.

39 P. de Tarso Garcia, T. M. G. Cardoso, C. D. Garcia, E. Carrilho and W. K. T. Coltro, RSC Adv., 2014, 4, 37637-37644.

40 E. Carrilho, A. W. Martinez and G. M. Whitesides, Anal. Chem., 2009, 81, 7091-7095.

41 M. Lehto, Paraffin Actuators in Microfluidic Systems, PhD thesis, Acta Universitatis Upsaliensis, 2007.

42 L. Y. Yeo, H. C. Chang, P. P. Chan and J. R. Friend, Small, 2011, 7, 12-48.

43 D. J. Guckenberger, E. Berthier and D. J. Beebe, J. Lab. Autom., 2015, 20, 146-153.

44 B. Li, L. Yu, J. Qi, L. Fu, P. Zhang and L. Chen, Anal. Chem., 2017, 89, 5707-5712.

45 A. W. Martinez, S. T. Phillips, G. M. Whitesides and E. Carrilho, Anal. Chem., 2010, 82, 3-10.

46 X. Chen, J. Chen, F. Wang, X. Xiang, M. Luo, X. Ji and Z. He, Biosens. Bioelectron., 2012, 35, 363-368.

47 S. H. Baek, C. Park, J. Jeon and S. Park, Biosensors, 2020, 10, 187.

48 C.-Y. Hou, L.-M. Fu, W.-J. Ju and P.-Y. Wu, Chem. Eng. J., 2020, 398, 125573.

49 Y. Zhang, Y.-L. Li, S.-H. Cui, C.-Y. Wen, P. Li, J.-F. Yu, S.-M. Tang and J.-B. Zeng, J. Anal. Test., 2021, 5, 11-18.

50 W. Zhao, J. C. Lam, W. Chiuman, M. A. Brook and Y. Li, Small, 2008, 4, 810-816.

51 Q. H. Nguyen and M. I. Kim, TrAC, Trends Anal. Chem., 2020, 116038.

52 A. Jafari, A. Emami and B. Ashtari, Mater. Sci. Eng., C, 2021, 121, 111810.

53 K. Gerelbaatar, A. Tsogoo, R. Dashzeveg, N. Tsedev and E. O. Ganbold, Solid State Phenom., 2018, 271, 76-84.

54 Y. Wang and Y. Ni, Talanta, 2014, 119, 320-330.

55 N. Chen, H. Liu, Y. Zhang, Z. Zhou, W. Fan, G. Yu, Z. Shen and A. Wu, Sens. Actuators, B, 2018, 255, 3093-3101.

56 X. Sun, R. Liu, Q. Liu, Q. Fei, G. Feng, H. Shan and Y. Huan, Sens. Actuators, B, 2018, 260, 998-1003.

57 A. Kumar, A. Hens, R. K. Arun, M. Chatterjee, K. Mahato, K. Layek and N. Chanda, Analyst, 2015, 140, 1817-1821.

58 S. Kumar, P. Bhushan and S. Bhattacharya, Anal. Methods, 2016, 8, 6965-6973.

59 B. S. Takale, M. Bao and Y. Yamamoto, Org. Biomol. Chem., 2014, 12, 2005-2027.

60 V. Vinoth, J. J. Wu and S. Anandan, Anal. Methods, 2016, 8, 4379-4390.

61 S. Bhattacharjee, J. Controlled Release, 2016, 235, 337-351. 
62 M. Havrdova, K. Polakova, J. Skopalik, M. Vujtek, A. Mokdad, M. Homolkova, J. Tucek, J. Nebesarova and R. Zboril, Micron, 2014, 67, 149-154.

63 R. White and A. Owens, J. Forensic Sci., 1987, 32, 1595-1603. 64 R. Egerton, P. Li and M. Malac, Micron, 2004, 35, 399-409.

65 C. M. Hoo, N. Starostin, P. West and M. L. Mecartney, J. Nanopart. Res., 2008, 10, 89-96.

66 A. Saeb, A. S. Alshammari, H. Al-Brahim and K. A. AlRubeaan, Sci. World J., 2014, 2014, 704708.

67 F. Farshchi, A. Saadati, N. Fathi, M. Hasanzadeh and M. Samiei, Anal. Methods, 2021, 13, 1286-1294.

68 V. V. Apyari, V. V. Arkhipova, A. I. Isachenko, P. A. Volkov, S. G. Dmitrienko and I. I. Torocheshnikova, Sens. Actuators, B, 2018, 260, 953-961.

69 M. R. Kateshiya, G. George, J. V. Rohit, N. I. Malek and S. K. Kailasa, Microchem. J., 2020, 158, 105212.

70 L. M. Fischer, M. Tenje, A. R. Heiskanen and N. Masuda, Microelectron. Eng., 2009, 86, 1282-1285.

71 W. Patungwasa and J. H. Hodak, Mater. Chem. Phys., 2008, 108, 45-54.
72 R. Cao-Milán and L. M. Liz-Marzán, Expert Opin. Drug Delivery, 2014, 11, 741-752.

73 A. Mobed, F. Kohansal, A. Ahmadalipour, M. Hasanzadeh and F. Zargari, Anal. Methods, 2021, 13, 311-321.

74 T. Ghosh, P. Sarkar and A. P. Turner, Bioelectrochemistry, 2015, 102, 1-9.

75 S. Verma, J. Choudhary, K. P. Singh, P. Chandra and S. P. Singh, Int. J. Biol. Macromol., 2019, 130, 333-341.

76 Y. Wang, Y. Yang, W. Liu, F. Ding, Q. Zhao, P. Zou, X. Wang and H. Rao, Microchim. Acta, 2018, 185, 1-9.

77 Q. Yan, N. Zhi, L. Yang, G. Xu, Q. Feng, Q. Zhang and S. Sun, Sci. Rep., 2020, 10, 1-10.

78 Q. Lu, J. Deng, Y. Hou, H. Wang, H. Li and Y. Zhang, Chem. Commun., 2015, 51, 12251-12253.

79 D. Yao, A. G. Vlessidis and N. P. Evmiridis, Anal. Chim. Acta, 2003, 478, 23-30.

80 T. A. Burinaru, M. Avram, A. Avram, C. Marculescu, B. Tincu, V. Tुucureanu, A. Matei and M. Militaru, ACS Comb. Sci., 2018, 20, 107-126. 